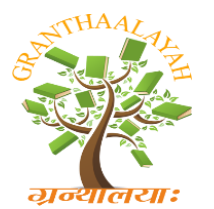

INTERNATIONAL JOURNAL OF RESEARCH GRANTHAALAYAH

A knowledge Repository

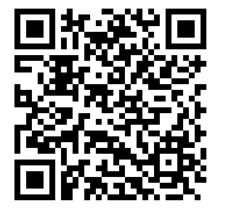

Management

\title{
PARADIGM SHIFT: ENGINEERING ARTIFICIAL INTELLIGENCE AND MANAGEMENT STRATEGIES FUSION
}

\author{
A. H. Harb ${ }^{1}$, AKA Abd Alhameed Abd Alhameed Alsayyid ${ }^{2}$ \\ ${ }^{1,2}$ Business Administration Department, The British University in Egypt, Sherouq City, EGYPT
}

\begin{abstract}
The purpose of this paper is to investigate management strategies that use Artificial Intelligence to perceive, capture, and process real-time data to predict and direct the performance of an enterprise. AI systems can account for errors in human judgment through computational processes that supersede the capabilities of human intelligence alone. Qualitative methodology was used to assess components such as fuzzy logic that can produce answers determined by multiple factors that can be integrated into a determinant solution. Quantifying the neurocircuitry of strategic management processes in tacticians will serve as the foundation for AI and management strategies engineering fusion. When AI is programmed to motivate, interact, and make judgements based upon statistical measurements, the fusion of AI and management engineering can increase efficiency and effectiveness in the attainment of organizational goals. Results demonstrated the application of AI planning can range from directing large-scale machinery overhaul procedures, spacecraft mission planning, emergency response, assembling test procedures for rocket launchers, and delivery truck scheduling. A thorough understanding AI for strategic management engineering fusion and its underlying concepts is a prerequisite to competitive advantage in a global market.
\end{abstract}

Keywords:

Artificial intelligence; strategic management; competitive advantage; engineering; fusion; cloud computing.

Cite This Article: A. H. Harb, and AKA Abd Alhameed Abd Alhameed Alsayyid, "PARADIGM SHIFT: ENGINEERING ARTIFICIAL INTELLIGENCE AND MANAGEMENT STRATEGIES FUSION" International Journal of Research - Granthaalayah, Vol. 4, No. 2 (2016): 1-16.

\section{INTRODUCTION}

The application of artificial intelligence for strategic management engineering fusion is a rapidly developing field. The fusion of artificial intelligence to software, hardware, and robotics for strategic management purposes alters the dynamic structure of an enterprise and increases efficiency while decreasing operational costs. Strategic managers must utilize concurrent developments in artificial intelligence research for engineering applications to remain 
competitive. An analytical understanding of the theoretical concepts that underlie artificial intelligence fusion to engineering applications will permit strategic managers to discern between emergent technologies and pre-existing constructs that have been modified. Applied concepts such as fuzzy logic, the Hick-Hyman law, and AI planning theory, inform strategic managers of how and why software encoded with AI can autonomously direct engineering applications.

The fusion of AI and strategic management has previously been utilized in power generation, infrastructure construction, and heavy manufacturing. These AI systems are based upon theoretical understandings of human behavior and thought that can be translated into algorithms and adaptable data sequences. Underlying theories such as fuzzy logic, the Hick-Hyman law, and AI planning theory, produce calculated decisions that can be strategically utilized for engineering purposes. The strategic decision-making process will serve as the standard by which AI will replicate the processes of learning and adaptive technology output (Edwards, 2015).

The goal of AI for strategic management engineering fusion is to transfer responsibility from human strategic managers to AI systems. Strategic managers may then address issues of conservation, political stability, and social welfare. AI systems can account for errors in human judgment through computational processes that supersede the capabilities of human intelligence alone. From military planning to the analysis of performances by professional athletes, AI systems have a range of applications that can be used for engineering analytics, land use management, and health, among others (Darrow, 2015). The fusion of AI for strategic management purposes will produce pragmatic solutions with predictive capabilities and serve society in the coming years (Brill et al., 2015).

Understanding behavioral psychology is essential to the design of artificial intelligence that can produce strategic decisions in a variety of circumstances. Making a choice is linearly related to the entropy of the possible alternatives (Schneider 2011). This concept is known as the HickHyman Law and it is one of the few laws which underlie behavioral psychology. By measuring the entropy of the distribution of reaction times, the relationship between reaction times and the available choices can be defined as logarithmic. Having a mathematical basis for behavior is the first step in producing artificial intelligence that can replicate behavior (Moscoso del Prado Martín, 2011).

The strength of synaptic connections shapes thought and decision-making processes and must be evaluated as a variable component in the production of AI for strategic management engineering fusion (Brockman \& Eagleman 2010). Spatial distribution, phosphorylation states, threedimensional arrangements of glia, and the affiliations of each aspect with neighboring proteins, can be modeled and evaluated for their role in the decision-making processes. Strategic management engineering fusion relies on systematic modes of analysis which will be modeled after the decision-making processes found in humans. The central nervous system and the endocrine system are also integral to human judgments and must be mapped accordingly for software input. These variables can be accounted for by the expansive capabilities of computer processors.

AI could be programmed to quickly assess data with multiple variables and then produce datadriven solutions for engineering firms under time constraints. Data must be integrated into AI 
systems so that the AI system can extract solutions from increasing aggregates of information. Solutions must contain quantitative goals or benchmarks that can be assessed by the AI system to determine success or failure (Aharony \& Noy 2009). The AI system will learn how to sort pertinent information from extraneous data with each successive trial. Instead of humans dwelling over large aggregates of data attempting to discern the most cost effective or efficient route of action, strategic managers could focus on the production of new solutions in thriving industries. The analysis of organizational goals, the production of multiple solutions, and the selection of a solution based on its symmetry to strategic management principles and engineering outcomes will serve firms who utilize AI for strategic management engineering fusion.

A variety of application domains across the engineering spectrum require the outcomes of AI planning. For any course of action selected by strategic managers there must be a preceding deliberation over the possible choices. Complex AI systems have been constructed to discern the most effective and efficient course of action in divergent circumstances (Wilkins 2014). AI planning must be task analyzed so that it may be modifiable for strategic management purposes. As AI planning develops the capacity to make choices aligned with strategic goals using historic data aggregates, AI planning will increase in efficiency and effectiveness. Planning is an explicit deliberation process that chooses and organizes actions by anticipating their outcomes (Laborie 2014). Under human supervision strategic managers prioritize actions and organize those actions into a plan carried by employees and workers. AI planning is the computational study of this deliberation process. The application of AI planning ranges from large-scale machinery overhaul procedures, spacecraft mission planning, emergency response, assembling test procedures for rocket launchers, and delivery truck scheduling (Gehlin, 2014).

Software models are integrated into AI planning systems as components by which these systems arrive at optimized outcomes. Sophisticated planning systems navigate different engineering contexts by mapping multidimensional space and generating a sequence of actions to lead to a goal state. These AI planning systems may possess domain-specific planning and domainindependent planning. Domain-specific planning uses distinct representations and techniques adapted to each problem for path and motion planning, perception planning, communication planning, and manipulation planning (Lan \& Toubia 2015). This may include navigating a robot through a three-dimensional space without bumping into other entities which can be imposed upon larger engineering purposes like air traffic controlling. Domain-independent planning uses generic representations and techniques, reducing the need for highly specific algorithms. The sources of domain analysis input vary and many engineers have sought to optimize the domain so that new inputs can be categorized to enhance reconfigurability (Figure 4.1). Domainindependent planning complements domain-specific planning in that it plans from first principles where novel situations are presented.

Studies have shown that an increased number of choices is linearly related to an increased reaction time (Psotta, 2014). The Hick-Hyman law has proven with accuracy that given specific parameters the reaction time to a stimulus with a select number of choices can be predicted for an individual. The variability of that individual's intelligence quotient is related to his or her information processing speed and has been correlated with variability in prediction accuracy (Woodley et al., 2013). This variability does not discard the application of the Hick-Hyman law in the behavioral sciences. The Hick-Hyman law can be used by engineering firms and the 
military to produce robotics infused with artificial intelligence capable of creating a specific number of distractions in a variety of locations that would produce a predetermined number of choices for an opponent. The Hick-Hyman law could then predict the amount of time that an opponent would need to produce a decision. This window of time could be used for military personnel to carry out a specific range of tasks before the opponent could produce a cohesive response or by engineering firms to produce budgets that estimate billable hours based on human behavior analytics.

Just as memory and processing power are added to computers, the mind is an adaptive system that adjusts to the processing power required of a task. The synthesis of human intelligence and computer processing is referred to as augmented intelligence. Augmented intelligence is used by corporations, governments, and individuals, to make possible strategic decisions that could not have been completed by human or machine alone (von Ahn 2013). The increased volatility in weather patterns due to climate change have led weather prediction experts to use augmented intelligence to heighten their predictive capabilities. In the last fifty years, predictive modeling for weather forecasts has gone from about twenty percent accuracy to now nearly eight percent accuracy (Brill et al. 2015). This fusion of human intelligence synthesized with computing power expands the range of possibilities available to engineering firms seeking strategic management solutions.

Analysis of behavior to produce AI for strategic management engineering fusion must encompass the possibility of equally intelligent choices. A variable that makes the imitation of human intelligence difficult is the concept of fuzzy logic. In fuzzy logic there are levels to the truth or the most efficient decision that can be made. Mathematically, fuzzy logic is represented by numbers of zero to ten. Fuzzy logic is the means by which computers can produce answers that are not simply yes or no, but rather determined by multiple components that must be integrated into a final solution. Fuzzy logic produces solutions by modeling the external environment using fuzzy sets and fuzzy rules. Fuzzy rules place specific parameters within which a decision can be made, while fuzzy sets quantify the range of choices and variables that can be taken into account. A fuzzy set is a set of related items which belong to that set in different degrees. Fuzzy logic avoids the need for rigid boundaries in data sets. Fuzzy sets permit AI systems to make decisions using imprecise quantities, similar to the human brain (Long, N, \& Meesad, 2014). Every strategic management decision contains high levels of uncertainty that need to be taken into account. For executives implementing AI into their automated buying systems, fuzzy logic can simulate human decision-making to produce strategic management decisions.

Cognitive computing, or the integration of humans and machines symbiotically producing solutions, incorporates AI for strategic management engineering fusion through the simulation of human thought process in a computerized model. Situations characterized by ambiguity can produce solutions by mimicking self-learning behaviors in humans. Using pattern recognition, data-mining, and natural language processing, cognitive computing draws from the strength of humans and computers (Galik \& Galikova 2015). The goal of cognitive computing is to produce an AI system that could accurately assess circumstances and supersede the limits of human cognition to produce data-driven solutions in heterogeneous frameworks. 
AI for strategic management engineering fusion has also found its way into cultural production. For every play in baseball there is data that explicates how and why that play was successful. If you mention number seven-hundred fifty-five, someone may say, "That is Hank Aaron's career home runs." If you mention sixty-one, someone may say, "Roger Maris hit sixty-one home runs in 1961." From televised ESPN programming to Major League Baseball stadiums in all major cities across the United States, measuring statistics is integral to analysis of baseball (Kornspan 2014). Fans, analysts, and entertainment program directors seek key statistics which include the speed of pitches, the distance of a hit ball, the time it takes for a baserunner to travel from one base to another, and the placement of each pitch relative to the batter. Data analysis drives player performance evaluations and informs managerial decisions.

For AI to effectively manage human endeavors it must interact with humans" "Darwinian" nature by tracking user's motions, vocal cues, and behavior patterns, to demonstrate sentience. This is controversial because humans begin to develop emotional attachments to digital, non-sentient entities that cannot reciprocate human emotions. This facet also implicates the potential for AI's role in hospice care, education, maternity studies, and human resource fields, where levels of sociable interaction can predict the success of an enterprise. AI systems can internalize expansive data regarding habits, choices, and the physiological preferences of their social counterparts. AI can be designed to statistically measure the productive capabilities of employees, thereby fulfilling the role of a strategic manager (Holzinger \& Maurer 1999).

The fusion of AI to engineering needs will produce solutions to problems that previously crippled the structure of global firms. Oil spills like Deepwater Horizon can be addressed more quickly and with greater efficiency when AIV systems are optimized to deal with the specific parameters of an oil spill. A proactive, AIV system that provides feedback of environmental conditions, safety monitoring, and intervention services, will increase the revenue of services and maximize efficiency for subsea engineering and construction companies.

\section{METHODS}

\section{FUZZY LOGIC}

Fuzzy rules take facts and determine to what degree the inputted information is true according to that rule. Rules use words and human concepts, rather than strict measurements. These rules can be combined in a process called inference, which can model the real world. Fuzzy logic is currently used to allow computers to mimic human decision-making. In neural networks it represents and captures input and output relationships to produce strategic management decisions fused with engineering applications. Like management, fuzzy logic learns on a trial and error basis, mimicking the characteristic of successful strategic managers (Edwards, 2015).

Engineering corporate decisions requires AI that can simulate high levels of variability and rapid change. For example, imagine the CEO of an international grocer is deciding on order quantities. Surely, she does not want to order low quantities that could be detrimental to efficient sales numbers. However, if she orders too much a great deal of the inventory may need to be inefficiently discarded. Furthermore, she must take into account the time of year, if there are any major holidays approaching, and what the weather will be like during the sales period (Cohen et 
al. 2015). Instead of classifying an item as buy or do not buy, strategic managers can use fuzzy logic to compute the degree of buying they want to impose on the AI system.

The use of fuzzy logic contrasts traditional logic theory where there is only true and false. Many decision support systems are based on fuzzy logic using interactive AI software based systems that support organizational goals. These systems help strategic managers compile data and select problems that must be dealt with to ensure the financial health of an organization (Alshibly, 2015). Strategic managers can input personal knowledge, comparative sales figures, and historical data to predict price movements in and intelligently generates a sell or buy signal to inform the leadership of how customers are perceiving organizational activity.

\section{AUGMENTED INTELLIGENCE}

Computer scientists and strategic management experts have sought to produce the most effective means for encoding human intelligence into AI systems. Programming techniques have spanned from logic programming, probabilistic searches, and classical searches (Whitson et al. 2015), to bayesian probabilities that continually incorporate new evidences from relevant data inputs (Huelsenbeck et al. 2001). Parallel analysis of various components, reinforcement learning, the ability to integrate several parameters, and choosing between competing models for the construction of objective priorities are of primary concern in the development of AI for strategic management engineering fusion.

Encoding the mind to be of use in strategic management for engineering fusion requires an understanding of the subtle thought processes of a strategic thinker. Japanese researchers performed a study which compared the brain activity of expert strategic tacticians to amateur strategic tacticians. With their brains monitored by an fMRI machine, the groups of tacticians were exposed to images of an active chess game for one second. The purpose of this brief image was to bypass the conscious portion of the brain's decision-making process to examine the subconscious role in strategic decision-making.

The researchers noticed that the precuneus portion of the brain was more active in the expert strategic tacticians compared to the amateurs strategic tacticians. The precuneus is integral to pattern recognition and visual-spatial reasoning. When both groups of tacticians were given the opportunity to make a subsequent move on the chessboard according to a one-second image of the chess game they had previously viewed, the caudate nucleus was highly active for the expert strategic decision-makers. The caudate nucleus is associated with learned response functions, habits, and long-term memory. The stronger the signal was in the caudate nucleus, the more likely the move selected by the expert was beneficial to win the chess match (Gillian et al. 2015). By mapping the neurocircuitry of the caudate nucleus and precuneus regions of the brain researchers can replicate the strategic management capacities of expert tacticians. This map can be transposed into software for engineering firms that can analyze real-time data to make intelligent decisions and predictions.

The San Francisco-based company, Quid, uses augmented intelligence to guide strategic management decisions for international engineering firms. Bio-analytics data, semantic analysis, neuro-linguistic programming, and network optimization, form streams of unstructured data that 
are filtered through the Quid analytics system. Applying a physic engine to obtain spatial resolution, the Quid Intelligence Platform creates visualizations that connect data and information from disparate resources. Quid AI software produces data landscapes (Figure 3.3) to make complex problems comprehensible for investors and innovative companies (Clegg, 2015). As these technologies are optimized for engineering fusion applications, strategic managers that utilize data landscapes like Quid will remain ahead of competitors.

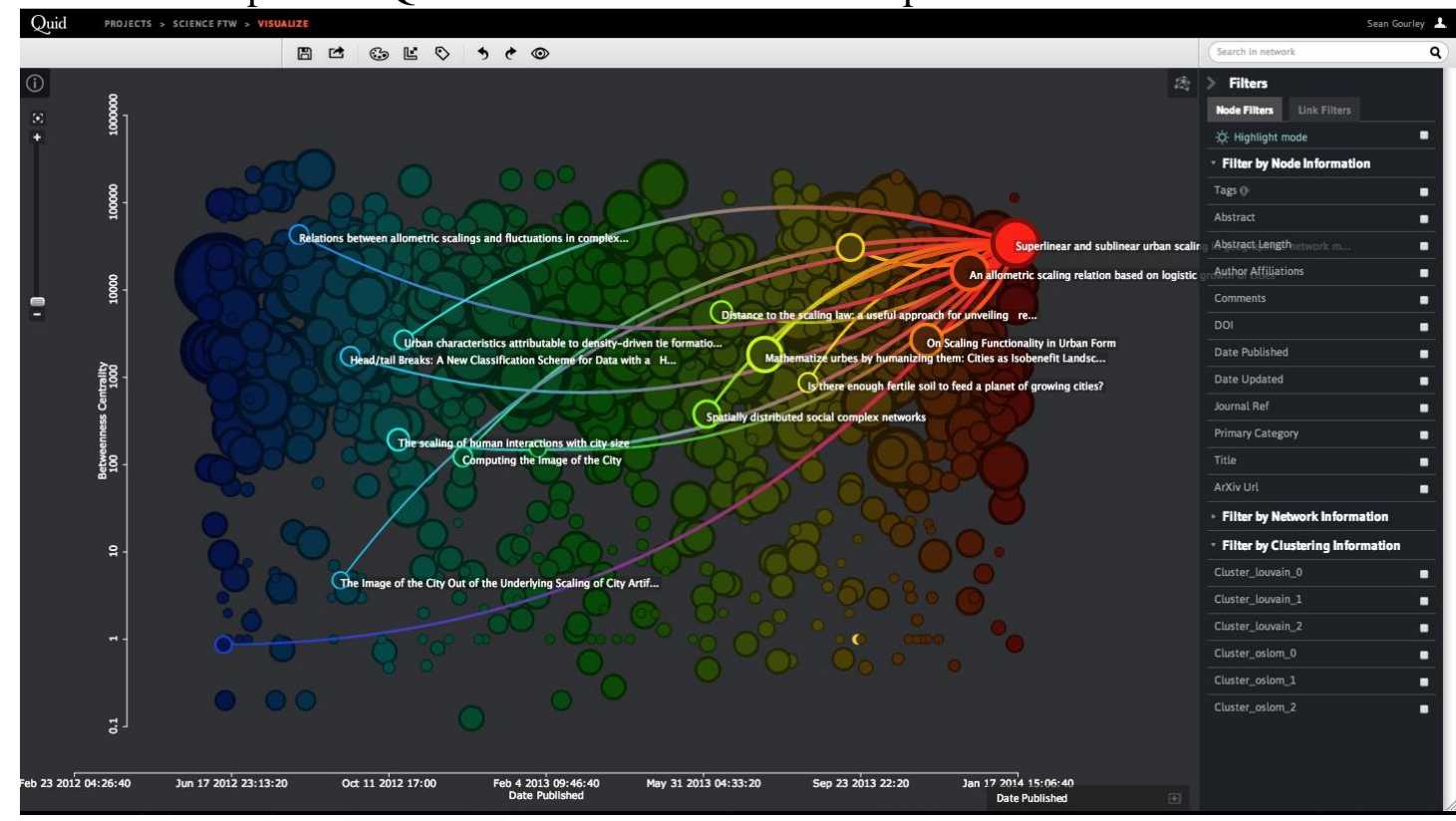

Figure 1: A physics engine is applied to streams of unstructured data filtered through the Quid analytics system to obtain spatial resolution of trends in data.

Rather than cluster keywords, Quid produces ideas that are at the periphery of data based on comparative filings, patents, journalism, and other disparate resources available in the public domain. Examining the data through a spatial resolution, strategic managers can understand market narratives and how strategies are playing out in the global economy. The visualization provides a geographic layout of data allowing strategic managers to make decisions based on intuitively aggregated data by AI software. Understanding the regions of technology that are heavily invested in can guide managers toward infusing their engineering practices with state of the art technology. This fusion of artificial intelligence and strategic management makes augmented intelligence a necessity for engineering firms.

The success of AI for strategic management engineering fusion will largely be determined by the ease of interaction AI systems have with their human counterparts.

\section{AI PLANNING}

AI planning is integral for explicit planning in a variety of contexts. When there is great risk potential, complex tasks, or collaborative and coordinated processes, AI planning can systematically determine outcomes and make strategic decisions. Using a generation of action sequences in robots, AI planning has been used in flexible assembly lines for the production of automobiles and boats. These robots are capable of selecting the correct fragment from an amalgamation of parts and successfully applying that fragment to the construction of a larger 
mechanism (Gerevini et al. 2009). AI planning can generate arm movement sequences that interact with the environment. Through the use of execution support facilities, STRIPS was capable of dealing with partial failures in planning by generating what was referred to as "macrobes" (Czerkawski \& Lyman 2015). STRIPS has proved foundational in the field of AI planning and new technologies are fusing strategic management principles with engineering applications to redefine the structure of engineering firms.

Challenges in this field include spatial issues and mechanical reasoning. AI planning can produce dynamic behaviors that may operate independent of human interference if these challenges are resolved. An example includes the reactive execution agent used to autonomously control the NASA Deep Space 1 spacecraft in its flight around a comet (Lambright 2015). AI planners have been used in search and rescue missions, electricity turbine overhauls, and experiment planning in molecular genetics. Artificial planning in dynamic situations will result from observations of real-world planning and execution which can be quantitatively analyzed and transposed into AI planning software. An ideal AI planning system would propose options to meet the stated objectives and either automate the selection process or refer to a human strategic manager for final deliberation.

\section{COGNITIVE COMPUTING}

IBM's SyNAPSE project, led Dharmendra Modha, is connecting seven IBM laboratories and four universities, integrating neuroscience, supercomputing, and nanotechnology, to create computer architecture that mimics the function, low power, small size, and real-time, of the human brain. Modern computers rely on symbolic, sequential nature which is likened to the left brain processes. The human brain is capable of parallel thinking, or the utilization of both hemispheres of the brain to solve conflicts. This SyNAPSE project will theoretically possess the capabilities of both hemispheres while allowing AI potential to seamlessly migrate between hemispheres. The neuron synaptic chips produced by IBM's SyNAPSE project are structured to process information using a network of over one million synthetic neurons (Alnajjar et al. 2008).

Researchers at the SyNAPSE project have mapped the largest diagram of a monkey's brain yet recorded (Figure 2). The bundles of radiant wire represent the impulse routes which pass by diffusions of neurotransmitters. These physical connections across hemispheres of the brain are mapped into visualization after being individually recorded into a spreadsheet. This comprehensive glimpse into the structure of a brain will demonstrate how the structure modulates dynamics. These dynamics give rise to behaviors and the visualization of these dynamics help scientists understand the brain's ability for sensation, cognition, perception, interaction, and action. Combining multiple sensory modalities while consuming less power than a light bulb and occupying less volume than a two-liter soda, the brain serves a model for highly efficient engineering. Mapping the brain to produce logarithms that imitate its functioning capabilities will create a shift from long sequential thinking to short parallel cognition (Ivanenko et. al, 2015). With short parallel cognition, AI for strategic management engineering fusion will give rise to machines that process real-time data and predict the performance of an enterprise. 

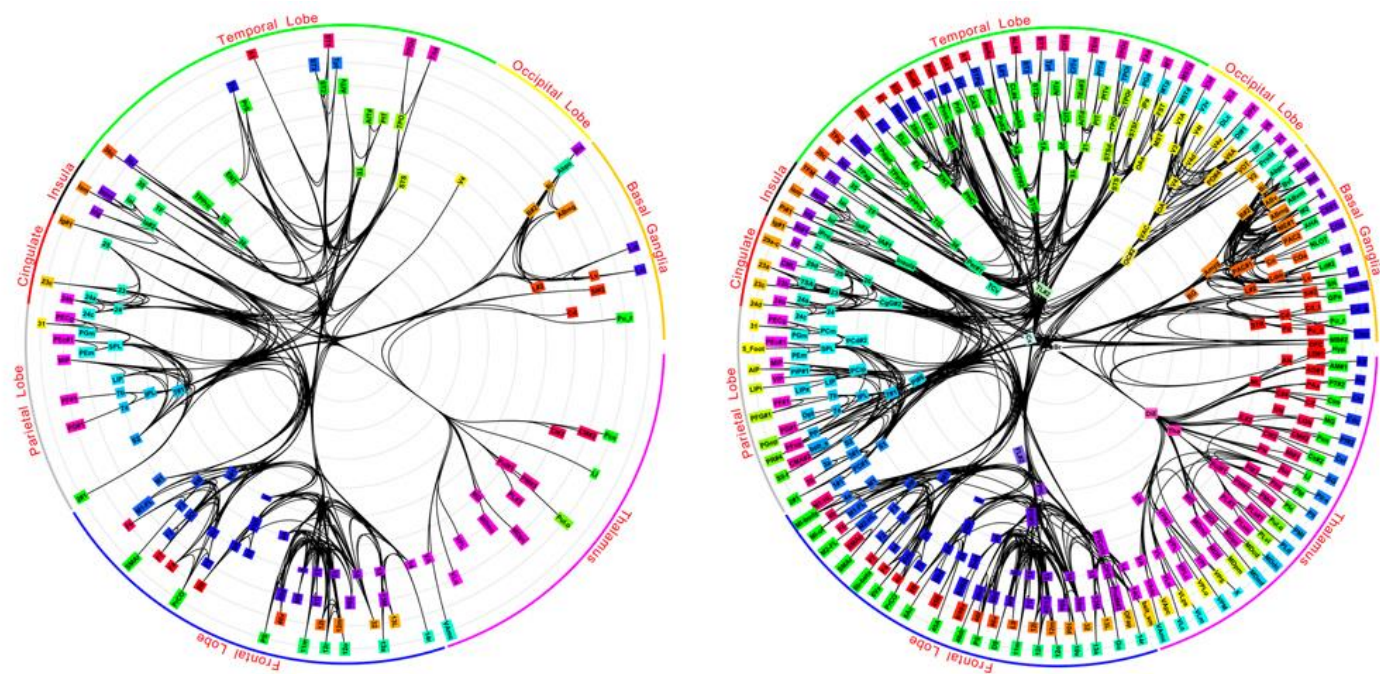

Figure 2: IBM's SyNAPSE project in which bundles of radiant wire represent the impulse routes which pass by diffusions of neurotransmitters.

Left: Neurosynaptic cognitive computing chip before meeting cognitive learning objective. Right: Neurosynaptic cognitive computing chip after meeting cognitive learning objective

IBM is connecting disparate technological elements and professionals across fields to produce AI for strategic management purposes. Fred Jelinek, an information theorist for IBM in the 1970s found that speech recognition systems would be improved not by linguists, but by engineers. These engineers were capable of reassessing the needs of speech recognition programs to alter the industry so that translation is based off data. Using a set of translation examples, automated speech recognition software produces potential translations. The more a speaker uses that device or system for speech recognition, the more their speech patterns are enrolled in the system, thus improving accuracy (Mohri et al. 2002). Google, Xbox, Siri, and Skype are just a few of the companies that employ deep learning to mediate automatic speech recognition systems.

\section{ENGINEERING FUSION}

The genomics community has produced data that can isolate changes in the genetic code that produce human traits and diseases (Brockman, 2010). Technology has been developed to compare genome sequences with the goal of isolating why certain humans are born with genetic disorders such as Fragile X syndrome, Down syndrome, and other disorders (Shaw et al. 2007). AI can intake genetic information, compare it with a database of similar and dissimilar genetic sequences, and predict the likelihood of genetic disorders and genetic traits that may appear in unborn children. Health systems can be engineered so that routine blood samples are analyzed to produce data on the predictability of a child inheriting certain genetic disorders. The J. Craig Venter Institute has produced bacterial chromosomes with more than " 582,000 nucleotides." Producing software that is capable of internalizing these large aggregates of data and subsequently making strategic management decisions will allow patients greater flexibility in their choices and improve national health. 


\section{FINDINGS}

Although the closed environment in which fuzzy logic operates cannot substitute the open environment in which humans produce decisions, engineers can easily adapt the parameters or fuzzy sets in which decisions are produced. There is a significant gap between the introduction of new variables to human leadership and those which are found in various data sources and computational systems. Engineering companies must demonstrate substantial accuracy to justify the transition of AI in place of strategic managers. Companies currently employ fuzzy logic for algorithmic trading and petroleum engineering in the oil and gas industry. Shell, ExxonMobil, Suncor, and Imperial Oil use fuzzy logic in deep water drilling, reservoir analysis, and petrophysics. Algorithms employing data from environmental landscapes are utilized to discover new oil and gas deposits daily. The most prevalent use of fuzzy logic is by Japanese companies such as Canon, Mitsubishi, IBM Japan, Toyota, and Nissan, in the design of products such as camcorders, banking systems, elevators, and a host of other products are dependent on this form of AI (Ning et al. 2015).

AI for strategic management engineering fusion can be assisted by the use of neural networks or deep learning (Hinton et al. 2006). Deep learning can be applied to interpret unstructured data, model high-level abstraction, and discern subtle evidences through the comparison of small bits of data extracted from large aggregates. Extracting meaningful attributes from input data, deep learning eliminates incorrect choices and systematically learns correct choices. This hierarchical learning model adapts to improve audio recognition and bioinformatics to produce higher levels of pattern recognition or CRM automation. Deep learning has been used in voice-recognition software and as these neural networks access larger aggregates of data their pattern-recognition accuracy improves (Shadiev et al. 2014). DeepFace, an application used by Facebook, uses spatial mapping to recognize faces with a baseline of 97.25 percent accuracy. DeepFace's neural network improves as user data confirms or refutes provided choices, thus meeting the qualification of adaptive. As deep learning adapts to identify the subtleties of language, different modalities such as speech, language, and vision require decoding the brain's 100,000 billion nodes and 100,000 trillion connections (Yu et al. 2015).

Researchers in Australia have applied AI software for strategic management of engineering weather preparation. This software uses pattern recognition algorithms that mine historic rainfall data to predict rainfall levels for up to three months in advance (Gillanders et al. 2011). To prepare for devastating floods scientists and researchers have devised AI for strategic management engineering fusion to produce solutions that can assist with migratory movements for residents in areas affected by tropical storms and monsoons. Quickly accessing an amalgamation of data on weather patterns across intersecting regions requires sourcing from radars, satellites, weather balloons, oceanic research vessels, and aircrafts designed to penetrate mercurial environments. This has challenged researchers, scientists, and computer programmers to produce AI which can rapidly gather data from a multiplicity of sources and disseminate it in a manner that will lead to strategic decisions that will reduce the destructive effects of global climate change.

As genes are sequenced and mapped they can also be engineered to alter a person's susceptibility to disease or hair color. Sequencing all 20,000 genes of the double helix requires an analysis of 
the adenine, thymine, cytosine, and guanine that compose the nucleotides. Automating this process collecting a teaspoon of blood or saliva will allow engineers to alter chromosomes that house different genes and ensure patient health. The scale of this engineering is still being developed to provide an inexpensive treatment option for patients (Lee et al. 2010). Composite genomes made of multiple people's DNA have been formed and AI automation systems will be able to assess and alter genetic engineering for strategic management purposes of health and disease eradication.

\section{DISCUSSION}

AI for strategic management engineering fusion can serve humanity and business in a profound manner. In a world ravaged by poverty, war, and disease, AI can overcome errors in human judgment by effectively managing resources. AI must be designed to perceive, discern, capture, and process real-time data feeds, both internally and externally to strategically manage resources and predict the performance of global enterprises. Instead of sifting through complex data aggregates with the goal of discerning the most efficient route of action, AI can be engineered to produce a composite of data-driven solutions that strategic managers can select or automate.

Quantifying the neurocircuitry of strategic management processes in expert tacticians will serve as the foundation for AI and engineering fusion. The visual cortex recognizes patterns and produces strategic decisions based upon these patterns. An adaptable AI system will analyze environmental patterns, materials, and other variables to make choices based upon parameters programmed through fuzzy logic. Just as the precuneus intuitively analyzes an abstract pattern to produce a strategic decision, robots constructed with AI software have engineered dynamic processes such as flexible assembly lines for the production of vehicles by selecting the correct parts from a variety of choices (Cavanna et al. 2006). The caudate nucleus exhibits a learned response that is sealed in the long-term memory and guides the subconscious processes. Using the fMRI images of the subconscious processes that guide expert tacticians as blueprints, AI systems regenerate these processes into software that receives real-time data and produces optimal decisions.

The pragmatic integration of AI for strategic management engineering fusion will be dependent upon a synthesis of available technologies. Strategic managers must understand what AI mechanisms will be most applicable to perceive, capture, and process real-time data to predict the performance of an enterprise. Enterprises need AI systems that can be matched to the data they are collecting, analyzing, and basing decisions upon. Cloud computing is an excellent example of a system that can be modified and engineered to shape organizational structure, reduce costs, and allow strategic managers to focus on solutions for customers. Some AI systems may be managed through cloud computing, while others will function outside the parameters of human interference. Comprehending the technology that governs AI decisionmaking processes will allow strategic managers to effectively orchestrate AI technology for dynamic engineering fusion.

Advanced analytics are used to discover undervalued talent, pursue players in free agency, and determine contract values. The relationship between advanced baseball analytics and strategic management decisions will improve as technology encompasses the physics involved with 
baseball performance evaluations. Statcast, which was announced at the MIT Sloan Conference by MLB Advanced Media, is the first use of AI to generate data that would not be available by human means alone. Through an amalgamation of cameras and radar measurements activated to track real-time data, Statcast captures the dynamic movements of players, objects, and motion within the frame of reference. Statcast is installed in all thirty of MLB's ballparks and provides a stream of data put to practical use by scouts, managers, and television analysts. Radar technology and optical tracking technology are based on technologies used to track space shuttle and missile launches to determine if debris was falling off. Statcast does the same for baseballs being thrown over one-hundred miles per hour and hit at even faster speeds with a high level of precision (Darrow 2015). The radar technology is effective at tracking the ball, while the optical tracking follows players in a large space. The two systems work symbiotically to create a threedimensional picture of what is happening in every single play. Statcast can track the baseball at 40,000 hertz (40,000 frames per second) and the optical tracking system tracks at twenty-five hertz, matching the visual capability of a broadcast.

Within one minute of a home run being hit, Statcast can produce the hitting metrics which include the exit velocity and projected home run distance. Feeding a replay through a Statcast tape machine allows for incredible visual acuity that indicates what speed the ball was hit, how far the ball was hit, and what angle the bat was positioned at when the ball was impacted (Blum 2015). The data is provided to each baseball team the following day for competitive purposes and strategic management decisions. Statcast can inform teams of whether or not a player is performing to the same capacity after an injury has occurred, to determine what players of value are available in the market, and what the value of an individual's contract should be. Statcast is a quantitative measurement of skills that can be used by managers, players, and fans to comprehensively engage in analysis and decision-making. AI has the capacity to engineer culture, health, and construction when automated systems are applied correctly for the purpose of analysis and decision making.

AI for strategic management engineering fusion must be run on shared data centers. Cloud computing is the most applicable technology to facilitate the integration of AI for strategic management purposes. Fusing advanced software and hardware with online hosting, cloud computing eliminates the need for servers, storage, and a technical team. Businesses can customize their applications without a need to hire an IT team or purchase more physical servers. Enterprise cloud computing is based on a multi-tenancy architecture which allows one application to be shared by multiple businesses. Each business can customize that application to their specific strategic management needs. Like an office building where each business has its own office space while sharing infrastructure and service's needs, cloud computing applications are elastic and provide users with the ability to remotely control AI systems.

As cloud computing applications are automatically upgraded, they receive new security features and performance enhancements will strategically allow them to gain a competitive edge. Instead of purchasing software and servers, the cost of utilizing an enterprise cloud computing system is based on a monthly subscription (Choudhary \& Vithayathil 2013). There are three levels to cloud computing: infrastructure, platform, and application. Cloud hosting is facilitated in the infrastructure. For companies experiencing rapid growth, cloud computing offers pre-existing infrastructures designed to handle that growth. Before cloud computing existed a company 
would need to host their website on a server. With increased network traffic the company would need to purchase more servers to handle an increased demand. Hundreds of thousands of companies still utilize this outdated and costly model of business.

The fusion of cloud computing for strategic management purposes eliminates the need for servers and costly IT personal. By hosting an interactive website on the cloud, a business can immediately scale up its computing power to match customer demands. If traffic reduces, servers can be released back into the cloud to reduce costs. The utilization of cloud computing is a major strategic advantage for businesses that successfully integrate this form of AI into their dynamic structure. Instant access to powerful servers allows companies to focus on engineering creative solutions, rather than purchasing the hardware and software necessary to begin new ventures. Gmail, Sales Force, and Netflix are examples of cloud computing serving as the infrastructure for an agile business model.

The strategic advantages that cloud computing has over the use of hardware and software are its scalability, its instant implementation, and its monetary benefits. Scalability refers to the number of servers that are necessary to host an online initiative. Whether it be one or one-hundred servers, cloud hosting allows management to easily increase or decrease the number of servers necessary to successfully engineer a project. The instant implementation of new servers for hosting the fluctuating needs of a business is the second advantage that allows cloud computing used by strategic management to reduce costs and increase power. Because businesses only pay for the hosting space they use, cloud computing eliminates the need for expensive hardware and software (Ghilic-Micu et al. 2014).

Applications in cloud computing can be modified to perceive, capture, and process real-time data to predict the performance of an enterprise. Strategic managers must focus on cost, competition, and customers. The utilization of cloud computing empirically reduces costs, aligns a business to compete with the likes of Netflix, Google, and Sales Force, and provides customers with a competent model for delivery of services. Strategic managers must actively intervene in a company's structure when potential is detected. Cloud computing's diverse advantages automate the function of IT and produce a competitive advantage that will lead to a sustained industry growth rate.

AI that produces higher predictability for ascertaining the relationship between events not discernable to human perception can guide strategic choices and tactics. Artificial intelligence has been successfully used in large-scale machinery overhaul procedures, spacecraft mission planning, emergency response, assembling test procedures for rocket launchers, delivery truck scheduling, and a host of other purposes. As AI technology is disseminated strategic managers will be required to discern between technologies. A thorough understanding AI for strategic management engineering fusion and its underlying concepts is a prerequisite to competitive advantage in a global market.

\section{CONCLUSIONS \& RECOMMENDATIONS}

Consistent, logical, and fair geopolitical policy is necessary for societies that are divided by borders and historical feuds. A 2014 study on coastal management and planning demonstrates 
that land use change threatens the sustainability of ecological environments worldwide. Human expansion and economic growth are cited as primary reasons for the rate of " $1.3 \mathrm{million} / \mathrm{ha} / \mathrm{year}$ " (FAO 2010). From agricultural demands to the relocation of populations for access to resources, strategic management of coastal resources and planning for future population requirements is debated by scientists, policy makers, and international focus groups. The emergence of land use models that employ AI have grown increasingly useful with rapid climate change and global migration patterns. These models predict changes in coastal migration and land use behavior that can guide strategic managers to engineer human populations toward efficient and effective conservation changes.

The evolution of land-use models, beginning with the 1950's models that used 'statistic and deterministic' principles for urban and transportation modeling, to the current fifth generation of model which utilizes AI technology such as "artificial life, intelligent stochastic simulation models, evolutionary computing, spatial DNA, and knowledge-based intelligent systems," have given rise to new possibilities which can alter the course of strategic management and population engineering. (Wu and Silva, 2010). AI systems must account transportation systems, capital investments, and land inputs. With this array of data, automating solutions requires the AI system to predict how micro-choices produce macro results. The immensity of variables can be difficult to account for, but as AI systems are fused to address global issues, the production of solutions will rival the variables that seek to undermine them.

The application of AI planning systems to engineering fusions such as NASA's spacecraft missions predict a diffusion of AI planning technology into private enterprises. The transfer of AI planning to engineering applications to be used by strategic managers will capture real-time data and predict the performance of an enterprise. The AI planning system, Optimum-AIV, was used for Ariane assembly, integration, and testing (Aarup et al. 1992). AI planning systems are used for telecommand of meteorological satellites and autonomous spacecrafts. This has become increasingly important as offshore drilling has expanded into more remote locations throughout the globe. Operators are seeking autonomous AI systems to survey, manage, and intervene in deep sea drilling missions. Subsea 7, a leading engineering and construction company has collaborated with SeeByte to produce an AIV to operate directly from a host facility or from infield support vessels. These unmanned underwater vehicles will revolutionize Life-of-Field projects as they carry an array of onboard navigation tools and sensors that can last up to 24 hours independent of charging.

When AI is programmed to motivate, interact, and make judgments based upon statistical measurements of socially engineered AI devices, the fusion of AI and social engineering can lead humans towards increased efficiency and effectiveness in the attainment of organizational goals.

\section{ACKNOWLEDGEMENTS}

This research was partially supported by Irahmssa Corporation. I thank many of my good friends and colleagues from University of Washington, University of New York, University of South California, and University of South Florida who provided insight and expertise that greatly assisted in shaping and directing the research. 
I thank Dr. Tarek Ali, Associate Professor, British University in Egypt for valuable comments that wkeenly guided concept development and greatly improved the manuscript.

I would also like to show my deepest gratitude to Mohamed H. Abdoun, Research Assistant, Irahmssa Corporation for his tireless and relentless support throughout the construction of this paper. This paper would be nearly impossible without his commitment and support.

\section{REFERENCES}

[1] Aharony, J., \& Noy, E. Corporate long-range quantitative goals: profit or growth?. The Journal Wealth Management, 12(1), 2009, 75-88.

[2] Alnajjar, F., Zin, I. B. M., \& Murase, K. A Spiking Neural Network with dynamic memory for a real autonomous mobile robot in dynamic environment. Neural Networks, 2008. IJCNN 2008.

[3] Alshibly, H. H. Investigating decision support system (DSS) success: a partial least squares structural equation modeling approach. Journal Of Business Studies Quarterly, 6(4), 2015, 56-77.

[4] Aarup, M., Arentoft, M., Parrod, Y., Stader, J., Stokes, I., \& Vadon, H. OPTIMUM-AIV: a knowledge-based planning and scheduling system for spacecraft AIV. University of Edinburgh, Artificial Intelligence Applications Institute, 1992, 4-17.

[5] Basl, J. The ethics of creating artificial consciousness. American Philosophical Association Newsletters: Philosophy and Computers 13.1, 2013, 25-30.

[6] Berlatsky, N. (2015). Artificial intelligence. Detroit: Greenhaven, 2015, 48-75.

[7] Binner, J. M., Kendall, G., \& Chen, S. Applications of artificial intelligence in finance and economics. Amsterdam: Elsevier JAI, 2004, 143-156.

[8] Blum, R. (2015). Data deluge: mlb rolls out statcast analytics on tuesday. Points of View Reference Center, EBSCOhost, 2015, 117-128.

[9] Brill, K. F., Fracasso, A. R., \& Bailey, C. M. Applying a divisive clustering algorithm to a large ensemble for medium-range forecasting at the weather prediction center. Weather \& Forecasting, 30(4), 2015, 873-891.

[10] Brockman, J. This will change everything: ideas that will shape the future. HarperCollins: New York, 2010, 67-75.

[11] Carrero, R., Navas, F., Malvárez, G., \& Guisado-Pintado, E. Artificial intelligence-based models to simulate land-use change around an estuary. Journal Of Coastal Research, 70, 2014, 414-419.

[12] Cavanna, A. E., \& Trimble, M. R. The precuneus: a review of its functional anatomy and behavioural correlates. Brain, 129(3), 2006, 564-583.

[13] Choudhary, V., \& Vithayathil, J. The Impact of Cloud Computing: Should the IT Department Be Organized as a Cost Center or a Profit Center?. Journal Of Management Information Systems, 30(2), 2013, 67-100.

[14] Clegg, D. Evolving data warehouse and bi architectures: the big data challenge. Business Intelligence Journal, 20(1), 2015, 19.

[15] Cohen, N., and Ilieva, R. Transitioning the food system: a strategic practice management approach for cities. Environmental Innovation And Societal Transitions Sciencedirect, 2015, 76-89. 
[16] Czerkawski, B., \& Lyman, E. Exploring Issues About Computational Thinking in Higher Education.Techtrends: Linking Research \& Practice To Improve Learning, 59(2), 2015, 57.

[17] Galik, S., and Galikova, S. Influence of the internet on the cognitive abilities of man: phenomenological and hermeneutical approach. Communication Today, 6(1), 2015, 415.

[18] Gerevini, A. E., Haslum, P., Long, D., Saetti, A., \& Dimopoulos, Y. Deterministic planning in the fifth international planning competition: PDDL3 and experimental evaluation of the planners. Artificial Intelligence, 173(5), 2009, 619-668.

[19] Higuchi, T., \& Troutt, M. D. Dynamic simulation of the supply chain for a short life cycle product-Lessons from the Tamagotchi case. Computers \& Operations Research, 31(7), 2004, 1097-1114.

[20] Hinton, G. E., Osindero, S., \& Teh, Y. A fast learning algorithm for deep belief nets. Neural computation, 18(7), 2006, 1527-1554.

[21] Huelsenbeck, J. P., \& Ronquist, F. MRBAYES: Bayesian inference of phylogenetic trees. Bioinformatics, 17(8), 2011, 754-755.

[22] Lan, L., \& Toubia, O. Improving online idea generation platforms and customizing the task structure on the basis of consumers' domain-specific knowledge. Journal Of Marketing, 79(5), 2015, 100-114.

[23] Long, N., and Meesad, P. An optimal design for type-2 fuzzy logic system using hybrid of chaos firefly algorithm and genetic algorithm and its application to sea level prediction. Journal Of Intelligent \& Fuzzy Systems, 27, 3, 2014, 1335-1346, Business Source Complete. EBSCOhost.

[24] Moscoso del Prado Martín, F. Macroscopic thermodynamics of reaction times. Journal Of Mathematical Psychology, 2011, 55302-319.

[25] Ning, L., Martínez, J., \& Hernández Díaz, V. The balanced cross-layer design routing algorithm in wireless sensor networks using fuzzy logic. Sensors (14248220), 15(8), 2015, 19541. 